\title{
Simultaneous three-channel signal detection: Performance and criterion as a function of order of report
}

\author{
LAWRENCE D. POHLMANN \\ Air Force Human Resources Laboratory, Williams AFB, Arizona 85224 \\ and \\ ROBERT D. SORKIN \\ Purdue University, Lafayette, Indiana 47907
}

\begin{abstract}
Performance on a task requiring detection of three sinusoidal signals occurring simultaneously and independently was compared to single-channel performance. The signal frequencies were 500 , 810 , and $1,320 \mathrm{~Hz}$, and all signals were presented monaurally in noise. The data for each channel were analyzed conditional on the stimulus-response events occurring in the remaining channels and conditional on the order of report. The decrement in performance in any channel in the three-channel task was found to increase as the number of signals and/or "yes" responses present in the remaining channels increased. Increasing decrements and criterion shifts were noted conditional on the order of report. The data are consistent with a model attributing the decrements in multichannel listening tasks to the later or "cognitive" stages of processing rather than to the earlier "perceptual" processing stages.
\end{abstract}

When an observer is required to simultaneously perform more than a single auditory perception task, he usually performs less efficiently than when he is allowed to do the same tasks individually. The magnitude and nature of the resulting performance decrements has long been of major theoretical and experimental interest. While much of the earlier research in this area concerned the monitoring of speech messages presented simultaneously to the two earphone channels (Broadbent, 1958; Moray, 1959; Treisman \& Geffen, 1967), more recent research efforts have employed less complex signals (Lindsay, Taylor, \& Forbes, 1968; Moray, 1970). Although a variety of paradigms may be used to study what may be collectively termed "multichannel processing," we have restricted our attention to and recently reported on a series of experiments in which observers must simultaneously perform two independent signal detection tasks (Gilliom \& Sorkin, 1974; Pastore \& Sorkin, 1972; Sorkin, Pastore, \& Pohlmann, 1972; Sorkin \& Pohlmann, 1973; Sorkin, Pohlmann, \& Gilliom, 1973; Sorkin, Pohlmann, \& Woods, 1976 , Note 1). In all of these studies, a consistent pattern of results has emerged. Small overall decrements have been found when performance on two-

A brief version of this report was presented at the 87th meeting of the Acoustical Society of America, New York, New York, April 1974. This research was partially supported by a National Science Foundation grant to R. D. Sorkin. The authors would like to thank Dr. Stanley Parkinson, Department of Psychology, Arizona State University, for a critical reading of the paper. channel tasks is compared to that obtained in singlechannel tasks. More interesting, however, has been the nature of these decrements. Performance in each channel in the two-channel tasks is critically dependent on the stimulus-response events in the other channel. The decrement for a given channel is largely restricted to those trials in which a signal and/or a "yes" ("signal present") response occurred in the other channel. On those trials when neither of these events has occurred, performance in the two-channel condition is essentially equal to single-channel performance. Similar results have recently been obtained in time-paced detection tasks (Moray, 1975), with alphanumeric signals (Ostry, Moray, \& Marks, Note 2) and with bisensory signals (Shiffrin \& Grantham, 1974).

The current study extends the paradigm and type of analysis employed to a three-channel task. Since we had found that observers could perform twochannel tasks with the same efficiency as singlechannel tasks as long as not more than one signal or signal-like event was present, we were interested in whether this ability could be extended to three frequencies. Although Lindsay (1970) found performance in $2 \mathrm{AFC}$ tasks to decrease systematically as the number of channels to be processed increased, our previous results suggested that only negligible decrements might occur in detection tasks on those trials when no more than one signal frequency was present. Note that although the stimuli for such trials are identical to those which might occur in an un- 
certain frequency detection task, the multichannel response requires not only detection of the signal but also identification of its frequency. While most uncertain frequency studies have required only detection, Green (1961) found that the additional requirements to identify the detected frequency produced only a small loss of efficiency. Lindsay's and Green's results together with our own lead us to expect performance for any given channel to decrease as the number of signals and/or "yes" responses present in the remaining channels increased.

Although previous studies have employed three (or four) channel tasks, these tasks have involved more than a single sensory modality or have involved stimulus or response dependencies (restrictions) (Lindsay, 1970; Lindsay, Taylor, \& Forbes, 1968; Shiffrin \& Grantham, 1974). For instance, Lindsay's stimuli were not independent and a fixed order of responses was required. It was thought that a threechannel task in which the stimuli varied along a single dimension and in which there were no a priori stimulus or response contingencies would provide information not available from these prior experiments using more restricted tasks. One possible source of such information might be the pattern of dependencies among the channels. In our two-channel studies, the performance decrements for each channel conditional on a "yes" or signal in the other channel tended to be nearly equal. However, with three frequencies, we expected that the performance decrements for the three channels might not be equal or symmetrical. For example, the decrement for a particular channel might depend not only on whether another signal was present but also on the stimulus parameters of the other signal. If the information arriving on different channels is susceptible to inaccurate recognition, we might expect performance to be disrupted more by a signal at a nearby frequency than by a more distant and presumably more distinct signal. Gilliom and Sorkin (1974) have proposed a processing model involving such a noisy recognition process. Stimulus confusions of a similar nature have been reported in the visual perception literature (Estes, 1972; Wolford \& Hollingsworth, 1974). It seemed reasonable to expect that these kinds of conditional performance asymmetries might occur in tasks involving similar dimensions in a single sensory modality and might not occur in tasks involving either dissimilar or cross-modality dimensions.

Since earlier multichannel studies in both audition and vision have suggested that information is lost or degraded during the output stages (Averbach \& Coriell, 1961; Moray, 1967; Moray, Bates, \& Barnet, 1965; Sperling, 1960), we were also interested in performance as a function of order of report. In multichannel discrimination tasks, Lindsay (1970) required subjects to respond to the attended dimensions in a specific order and found performance to decrease more for later responses. Lappin (1967) noted similar effects in a three-channel visual discrimination task. Darwin, Turvey, and Crowder (1972), Kinchla (1973) and Treisman and Rostron (1972), have also provided evidence that auditory information may be stored only briefly. To the extent that information is lost or degraded over time, we might expect performance decrements to increase for the later responses. However, if (1) the overall performance decrement is to systematically increase as a function of order of report, and (2) the decrements obtained when not more than one signal is present are to remain negligible, increasing decrements for the later response would only be predicted for those trials when more than one signal was present.

\section{METHOD}

Three female students with normal hearing served as paid observers. They were housed in a single walled Industrial Acoustics chamber inside an acoustically insulated room. The basic task was a single-interval yes-no task; trial events were controlled and recorded by a PDP-8/L computer. After approximately $20 \mathrm{~h}$ of practice devoted to choosing signal levels and running two-channel tasks, six to eight 100 -trial blocks were run each day.

Two types of conditions were run, single-channel conditions and a three-channel (frequency) condition. In the three-channel condition observers were required to make three separate yes-no responses to the possible occurrence of three sinusoidal signals, one at $500 \mathrm{~Hz}$, one at $810 \mathrm{~Hz}$, and one at $1,320 \mathrm{~Hz}$. The probability of signal occurrence at each signal frequency was .5 and was independent of the stimulus events at the other two frequencies. The signals were mixed and then gated on and off at positive zero crossings. All signals were $100 \mathrm{msec}$ in duration and were presented simultaneously during a single, visually marked, $100 \mathrm{msec}$ observation interval. Continuous broadband gaussian noise, bandlimited only by the headphones (TDH-39), was present at an overall level of $62 \mathrm{~dB}$ SPL. The signals and noise were both monaural.

Observers made their responses on a 2 by 3 matrix of buttons, the three "yes" buttons being in the top row and the columns representing the three frequencies in ascending order from left to right. Observers were allowed to make the three required responses in any order. A 2 by 3 matrix of lights was used to indicate to the observer which responses she had already made. After all three responses had been made, the same matrix indicated the correct responses. All observers worked individually and responded at their own pace. The observers were instructed to maximize the total number of correct responses and were informed of the total number correct as well as the number correct at each frequency after each block of trials. Observers were encouraged to avoid biases toward any single frequency.

In the single-channel conditions, a single fixed frequency was presented with probability of occurrence of .5 and only a single yes-no response was required. Signal levels were chosen to yield single-channel d's of approximately 2.0 and corresponded to E/No levels of $9.8,13.1$, and 16.8 for the $500-, 810$-, and $1,320-\mathrm{Hz}$ signals, respectively. All observers were run at the same signal levels. A session was devoted to running either the three singlefrequency conditions in some blocked random order or the threechannel condition. Six to eight single-channel sessions were inter- 
spersed with an equal number of three-channel sessions. All observers were given several days of practice on two-channel tasks $(500$ and $810 \mathrm{~Hz}$ ) prior to the collection of the reported threechannel data.

\section{RESULTS}

The detectabilities of the single-frequency conditions for each observer are shown at the top of Table 1. The entries are d's averaged across sessions and frequencies. Performance on the three-channel condition is shown in the lower parts of Table 1. The entries are shown as percentage decrements from the single-channel conditions averaged across sessions and frequencies. The initial analyses in the three-channel task involving assessing performance at each frequency independently - without regard to performance or stimulus-response events at the other two frequencies. When this overall (unconditional) performance is considered, all observers were found to exhibit substantial decrements in the three-channel task compared to the single-channel tasks.

The data for each observer and frequency were also analyzed conditional on the stimulus and/or response events in the other two channels. For example, for a given frequency, a hit and false alarm probability can be computed by considering only those trials in which there was a signal at one (and only one) of the other frequencies. It should be obvious that, for any given frequency, a variety of partitions, based on the stimulus-response events at the other two frequencies, may be made. The decrements resulting from some of these partitions are shown in Tables 1 and 2.

Note first the middle section of Table 1 . When no other signal was present-the line labeled "No Signal"-performance was only slightly lower than performance on the single-frequency control conditions. When a signal was present at only one other frequency-the line labeled "One Signal" - a larger

Table 1

Performance Averaged Across Frequencies for Each Observer Conditional on the Stimulus Events in the Other Two Channels

\begin{tabular}{lrrrr}
\hline & \multicolumn{4}{c}{ Observer } \\
\cline { 2 - 5 } & CK & BL & LS & Average \\
\hline Single Frequency* $^{*}$ & 2.22 & 2.19 & 2.13 & 2.18 \\
Three-Frequency Unconditional $^{* *}$ & 36 & 34 & 17 & 29 \\
No Signal & 17 & 9 & 14 & 13 \\
One Signal & 28 & 26 & 12 & 22 \\
Two Signals & 46 & 42 & 23 & 37 \\
One Signal Distant & 11 & 18 & 23 & 17 \\
One Signal Adjacent & 36 & 31 & 7 & 25 \\
Two Signals Adjacent and Distant & 34 & 36 & 27 & 32 \\
Two Signals Bracketing & 71 & 54 & 16 & 47 \\
\hline
\end{tabular}

*Single-frequency entries are d's.

* Three-channel entries are percentage decrements from singlechannel d's.
Table 2

Three-Channel Performance Averaged Across Frequencies for Each Observer Conditional on the Response Events in the Other Two Channels

\begin{tabular}{lcccc}
\hline & \multicolumn{4}{c}{ Observer } \\
\cline { 2 - 5 } & CK & BL & LS & Average \\
\hline Three-Frequency Unconditional* & 36 & 34 & 17 & 29 \\
No "yeses" & 25 & 19 & 14 & 19 \\
One "yes" & 33 & 36 & 10 & 26 \\
Two "yeses" & 53 & 56 & 28 & 46 \\
One "yes" Distant & 27 & 34 & 20 & 27 \\
One "yes" Adjacent & 38 & 26 & 4 & 23 \\
Two "yeses" Adjacent and Distant & 35 & 49 & 33 & 39 \\
Two "yeses" Bracketing & 90 & 70 & 18 & 59 \\
\hline
\end{tabular}

*Entries are percentage decrements from single-channel d's.

decrement was obtained. The largest decrements were obtained when signals were present at both of the other frequencies.

When one or both other signals were present, the size of the performance decrements tended to be inversely related to the frequency separation of the signals involved. These data are shown in the lower part of the table. Generally, the presence of other signals at nearer frequencies produced greater decrements. Thus, the largest decrements of allshown in the bottom line of the table-were obtained for the $810-\mathrm{Hz}$ channel when it was "bracketed" by the presence of both the 500 - and $1,320-\mathrm{Hz}$ signals.

Table 2 shows the results of partitioning on the basis of "yes" responses. The same general effects may be noted. Performance is inversely related to the number of "yes" responses at the other frequencies. The decrements conditional on "yes" responses were almost always larger than the decrements conditional on signal presence. Breaking down the effects of "yes" responses in relation to the frequency separation of the channels again shows that the largest decrements tend to occur for the middle channel when both other "yes" responses are made. However, effects when only one other "yes" response was present were not consistently related to frequency separation.

The amount of data collected was insufficient to completely assess the results of partitioning on both stimulus and response events. It was possible, however, to partition out the data for each channel given both hits or both correct rejections in the remaining channels. These data are shown in Table 3. Performance decrements were large given hits in the remaining channels and were very small or nonexistent given two correct rejections.

The results of an analysis of the data conditional on the order of report are interesting and are shown in Table 4. The entries are percentage decrements and $\beta$ s averaged across frequencies and conditional on response order. In performing the analyses, we 
Table 3

Three-Channel Performance Averaged Across Frequencies for Each Observer Conditional on Selected Stimulus-Response Events in the Other Two Channels

\begin{tabular}{lrrrc}
\hline & \multicolumn{4}{c}{ Observer } \\
\cline { 2 - 5 } & CK & BL & LS & Average \\
\hline Two Hits* & 52 & 53 & 31 & 45 \\
Two Correct Rejections & 5 & -15 & 4 & -2 \\
\hline
\end{tabular}

*Entries are percentage decrements from single-channel d's.

Table 4

Performance and Criterion Measures on the Three-Channel Task as a Function of Order of Report

\begin{tabular}{lrrrr}
\hline & \multicolumn{4}{c}{ Observer } \\
\cline { 3 - 5 } & CK & BL & LS & Average* $^{*}$ \\
\hline \% Decrement** & & & & \\
Response 1 & 34 & 29 & 10 & 19 \\
Response 2 & 52 & 36 & 15 & 25 \\
Response 3 & 21 & 49 & 36 & 42 \\
$\beta$ & & & & \\
Response 1 & 1.35 & .27 & .22 & \\
Response 2 & .93 & 1.32 & 1.44 & \\
Response 3 & 1.30 & 4.06 & 8.07 & \\
Single Frequency & .82 & 1.61 & 1.01 & \\
\hline
\end{tabular}

*Observers BL and LS only

**rom single-channel d's

noted two different observer response strategies. Two of the observers, B.L. and L.S., distributed their first and later responses over the three frequencies, while observer C.K. responded in the same fixed order on almost every trial-making the $500-\mathrm{Hz}$ response first, the $810-\mathrm{Hz}$ response next, and the $1,320-\mathrm{Hz}$ last. Accordingly, the table entries for observer C.K. are not averaged across frequencies as are the entries for the two randomly responding observers. Two findings arise out of comparisons of performance and the order of report for the data from the randomly responding observers B.L. and L.S. Not only was performance lower for later responses,but dramatic criterion shifts were noted. These two observers were drastically more liberal for the first responses and just as drastically more conservative for their third responses. This resulted from a very marked tendency for these two observers to make all of their "yes" responses prior to making any of the "no" responses. The percentage of "yes" responses as a function of order of report is shown in Table 5.

It would be interesting to partition the data on the basis of both the stimulus-response event and the order of report, but an extraordinary amount of data would be required to result in a reasonable number of trials in each cell. While simply running more trials may decrease the variance of probability estimates, a related problem arises when some of the matrix entries are zero. If an observer's first responses are all or nearly all "yes" for some frequency, that is, mostly hits and false alarms, the use of $d$ ' as a performance measure may be inappropriate. ${ }^{1}$

\section{DISCUSSION}

Before relating the data to any particular models, we will consider briefly the overall performance decrements. The overall decrements obtained in the present three-channel task were slightly larger than those obtained in our most closely related twochannel tasks. Previously, we had noted average two-channel decrements of $19 \%$ (Sorkin, Pohlmann, \& Gilliom, 1973) and 12\% (Sorkin, Pohlmann, \& Woods, Note 1). The two-channel task used as part of the training sequence for the present experiment yielded average decrements of only $6 \%$. While twochannel decrements in other less closely related experiments in the series have ranged from $5 \%$ to $50 \%$ for particular observers and conditions, performance for any observer in a given condition is generally quite stable. The size of the overall decrements in the current experiment compare reasonably to the average decrements (approximately 22\%) reported by Lindsay (1970) in multichannel discrimination tasks employing a $2 \mathrm{AFC}$ paradigm.

The conditional decrements again prove to be more interesting and serve to lead into a consideration of models. The type of interference noted in previous papers in the series was again replicated. The present data indicate that interference is produced when either a signal, a "yes" response, or both, are present in a remote channel. In addition, orderly decreases in performance were found as the number of remote signals present or the number of "yes" responses made for the remote channels increased. Naturally, since we are dealing with d's generally ranging in magnitude from 1.0 to over 2.0, there is a fairly high correlation between a signal present and a "yes" response. Thus, the noted effects may be due either to the presence of a signal or to some later stages of processing correlated with a "yes" response. Note that in the former case some kind of stimulus interference model is suggested, while the latter implies that models hypothesizing

Table 5

Percentage of "Yes" Responses as Function of Order of Report

\begin{tabular}{llcl}
\hline & \multicolumn{3}{c}{ Observer } \\
\cline { 2 - 4 } & CK & BL & LS \\
\hline Response 1 & 44 & 81 & 84 \\
Response 2 & 53 & 45 & 46 \\
Response 3 & 47 & 18 & 14 \\
Three-Channel Overall & 48 & 48 & 48 \\
Single-Channel & 52 & 47 & 51 \\
\hline
\end{tabular}


interference at later processing stages should be considered.

In an effort to separate the effects of signal presence from those involved in responding "yes," consider first their effects in combination. If both produce some interference independent of the other, we would certainly expect that the interference effect would increase when both are present and to be reduced when both are absent. That is, for any particular channel the most interference should occur when there is a correctly detected signal in both of the other channels. Similarly, the interference should be least when neither signal nor "yes" response has occurred. The data in Table 3 partially support this hypothesis. The decrement is least when both other channels contained correct rejections. In fact, there was some degree of facilitation for observer B.L. For this observer, detection in the three-channel task, given correct rejections in both remote channels, was better than single-channel detection performance. However, the interference given hits in both other channels appears to be no greater than that given two "yes" responses (average decrement $45 \%$ ). This would tend to support the notion that it is some aspect of the processing leading to a "yes" response that produces the interference.

Next, consider the conditional effects of signal presence. As with all previous experiments in the series, the present data, when considered alone, seem consistent with a simple cross-masking hypothesis; that is, the presence of a signal in an adjacent frequency region increases the level of internal noise present in the frequency region under consideration. The fact that the amount of interference tends to decrease with increasing frequency separation is consistent with this hypothesis. However, when the results of several experiments are considered, there is a great deal of evidence against a cross-masking hypothesis. From earlier experiments in the series, we have drawn the following conclusions:

(1) For the frequency separations involved, the decrements are much larger than would be predicted on the basis of pure tone masking data. Note also that, since the signals were being detected in noise, the remote signals added only small increments to the acoustic energy already present.

(2) The effect is not decreased when the signals are assigned to separate earphone channels.

(3) The interference effect is symmetrical across both channels, that is, decrements in both channels were essentially equal.

(4) The interference occurs when the "signal" is actually a gap in an otherwise continuous sinusoid (Gilliom \& Mills, 1974).

(5) Lastly, in a study in which the actual noisy inputs were recorded for each trial, the size of the interference effect was found to be uncorrelated with the amount of energy in the vicinity of the remote channel signal (Sorkin, Pohlmann, \& Woods, 1976).

Together, these findings are strong evidence against any model which attributes the interference to any early stage of signal processing. The decrements that are obtained conditional on signal presence may presumably be attributed to the correlation between signal presence and processing leading to a "yes" response.

The consideration of these later processing stage as the locus of the interference leads us into the discussion of the criterion effects noted in the order of report data. One of the mechanisms that has been proposed to account for the decrements obtained in multichannel performance is criterion variability. Treisman (1972) suggested that the decrements noted by Moray (1970) could be explained in terms of a variable criterion. Treisman hypothesized that the criterion on a given channel might vary across trials with the magnitude of the observation in a remote channel. Data supporting this hypothesis have been recently reported by Moray (1975). Such a strategy is to an observer's advantage only when the observer knows that there are signal occurrence contingencies as in Moray's tasks. Garner and Morton (1969) discuss some of the analysis problems associated with tasks employing nonindependent signals. As we have previously noted (Sorkin, Pohlmann, \& Gilliom, 1973), for an observer to make his criterion dependent on the magnitude of the observation in a remote channel in an independent signal task would not be optimal. This fact, however, does not rule out the possibility that performance decrements in multichannel tasks are indeed due to criterion variability. Two distinct hypotheses may be made. The first is that the necessity to monitor two or more channels somehow prevents the observer from maintaining a stable criterion for any given channel. Such a possibility would be difficult to verify since it requires trial-by-trial estimates of the observer's criteria. Requiring the observer to make rating decisions in a multichannel task and at the same time analyzing the trial by trial physical stimulus, as in Sorkin, Pohlmann, and Woods (1976), may allow assessment of trial-by-trial criterion variations. However, any variations that do occur may have been introduced by the rating procedure. McNicol (1975) has recently suggested that the requirement to hold several simultaneous criteria adds noise to the observer's decision process.

A second hypothesis is that the requirements of a multichannel task introduce systematic criterion shifts in spite of their nonoptimality. Although we have repeatedly searched for such shifts, the only previous consistency was in the Gilliom and Sorkin (1974) study. They noted some tendency for observers to make similar responses in both channels, either 
both "yes" or both "no" responses. This was taken as evidence for a noisy recognition process. The criterion shifts in the present experiment were much more dramatic for two of the observers. As the data in Table 4 indicate, these two observers exhibited a very liberal criterion for the first response and then became increasingly more conservative for the later responses. While the differences are very striking at first glance, they follow logically from the response strategy employed by these two observers.

Consider the predictions concerning criterion that would be made for the three-channel task if an observer (1) tries to maximize the number of correct responses and (2) makes all "yes" responses prior to making any "no" responses. The criterion for the first response is a function of the a priori probability that at least one signal is present and the probability that no signals are present. These two events occur with probabilities of .875 and .125 , respectively. Therefore, since a signal event is much more likely than a noise-alone trial, the initial criterion for responding "yes" should be liberal. In a similar manner, the criteria for the second and third responses may be computed. These shifting criterion predictions and the predicted probabilities of responding "yes" as a function of order report are shown in Table 6. A comparison of the obtained $\beta$ s and probabilities listed in Tables 4 and 5 shows that the predicted shifts are quite consistent with, but larger than, those actually obtained. If an observer delayed any "yes" responses to make a "no" first response on only a few trials, the predicted shifts would be nearer to the actual shifts. Thus, the obtained criterion shifts are easily explained in terms of the hypothesized response strategies. A few additional points may be noted. These kinds of shifts will go unnoticed unless performance is analyzed conditional on order of report. If the task is set up such that the three decisions required must be mapped into a single (eight-choice) response, one could not ascertain the criterion shifts and differences in response strategy we noted.

The individual differences in order of report strategy are not unlike those noted in other tasks requiring the simultaneous processing of stimuli (Parkinson, Knight, DeMaio, \& Connors, 1974). It is interesting to note that the fixed order observer in the current experiment exhibited neither criterion shifts nor performance decrement increases as a

Table 6

The Predicted $\beta$ and Percentage of "Yes" Responses as a Function of Order of Report

\begin{tabular}{lcc}
\hline & \% "Yes" & $\beta$ \\
\hline Response 1 & 87.5 & .14 \\
Response 2 & 50.0 & 1.00 \\
Response 3 & 12.5 & 7.00 \\
\hline
\end{tabular}

function of order of report. Of course, the response order is not necessarily the order in which the decisions were made. In fact, in the two-channel condition used as practice, observer C.K. showed both a decrease in performance and a criterion shift as a function of order of report. In the two-channel task, she made all single "yes" responses first. If two "yes" responses were required, the $500-\mathrm{Hz}$ response was always made first.

The current data are highly consistent with a model which places the locus of multichannel interference relatively late in the system. If we divide the processing required in multichannel tasks into the general categories of "perceptual processes" and "cognitive processes" (Shiffrin \& Grantham, 1974), the effect would be placed in the latter category. Perceptual processing may be assumed to end with the dumping of information into a short-term store. This information may be the outputs of several criticalband-like mechanisms. We might label as "cognitive processing" the remaining kinds of processes which must be carried out before the responses are executed. This includes operations like scanning, rehearsal, coding, and decisions. Since the information in the short-term store will be lost in fairly short order, performance should suffer as the complexity of the cognitive processing required increases.

Let us consider briefly what kinds of predictions such a model should make for the conditional data. First, we assume that the perceptual information is not altered by the requirement to monitor three channels, that is, the information available at each frequency when cognitive processing begins is no different in the three-channel task than in the respective single-channel tasks. While in the singlechannel task both the detection decision and the nondetection decision map directly into a response, in the three-channel task only the nondetection response is similarly determined-three "no" responses. Since the response is determined, presumably the amount of cognitive processing required is minimal. Thus, when no signals are present, a minimal performance decrement would be predicted, any actual decrement probably being associated with the increased likelihood of a false alarm. The likelihood of more than a single false alarm given no signals should be neglible.

A more complex decision process must be carried out if detection occurs. Assume that one of the inputs (outputs of one of the critical-band-like mechanisms) exceeds some critical preset level. The observer must decide in which channel criterion was exceeded-the recognition process. The process may be somewhat analogous to the processing of an interrupt by a computer, an analogy proposed by Gilliom and Sorkin (1974) for their interrupt-noisyrecognition model. In that model, the "interrupt" 
is the detection. Note that hypothesizing a passive perceptual system is tantamount to assuming that the "interrupt" is effectively not enabled until integration processes are somewhat complete. Positing that the largest input is processed first is consistent with the execution of "yes" responses first and with the result that the hit rates on the first response are greater than single-channel hit rates. While the first interrupt is being processed, information concerning the remaining channels is subject to decay and/or distortion caused by channel cross-talk (both possible provisions for individual performance differences). Thus, when a signal is present, an interrupt is likely; the processing of this interrupt, most likely leading to a "yes" response would result in performance decrements in the remaining channels. Note that "channel cross-talk" as used above refers to activity during the cognitive stages of processing, an effect somewhat different from simple cross-masking which might be expected to occur during perceptual processing.

When two of the inputs exceed criteria, the interrupt or recognition process must be executed twice, causing the remaining third channel to suffer even more and resulting in still larger decrements.

So far, only the conditional data concerning the number of signals and "yes" responses have been discussed. The asymmetries of the conditional data, that is, the differential interference effects corresponding to signal frequency relationships, are somewhat more complex. The model, as defined above, predicts no asymmetry for two-channel conditions, the obtained result. The model also predicts the increasing decrements as channels become more similar (Gilliom \& Sorkin, 1974) as the recognition process may be expected to take longer. However, in light of recent demonstrations of attentional effects, predictions based on any simple intuitive notions regarding signal confusability would probably be difficult to support. Several studies have recently demonstrated attentional effects (performance decrement asymmetries) in tasks when two independent signals may occur (Geschieder, Sager, \& Ruffolo, 1975; Shiffrin \& Grantham, 1974; Sorkin, Pohlmann, \& Woods, Note 1). To predict these results, additional revised assumptions concerning the recognition process are required. The premise that processing of the largest input is completed first must be revised. Although there are other alternatives, the computer analogy may be extended if the interrupt processor is assumed to include provision for processing priorities. The confusability of a signal with other signals may be one factor involved in priority assignment. In tasks like the current one, the possibility of complex interactions between confusability effects and attentional effects preclude definite predictions concerning asymmetry at this time.
In summary, a model comprising an independentchannel "perceptual" processor feeding into a "cognitive" processor susceptible to channel interactions seems to be supported by the current data. Such a model would, in Norman and Bobrow's (1975) terms, be resource-limited rather than datalimited. Several interesting issues concerning the general applicability of this type of model remain to be investigated. While analogies to an interruptlike process are appropriate for detection tasks, the applicability of the construct to more complex tasks as well as the nature of conditional performance in such complex tasks is yet to be determined.

\section{REFERENCE NOTES}

1. Sorkin, R. D., Pohlmann, L. D., \& Woods, D. D. Methodological aspects of two-channel signal detection: Listener strategy and the dedicated condition. Manuscript submitted for publication, 1976.

2. Ostry, D., Moray, N., \& Marks, G. Attention, practice, and semantic targets. Unpublished manuscript. 1975.

\section{REFERENCES}

Averbach, E., \& Coriell, A. S. Short term memory in vision. Bell System Technical Journal, 1961, 40, 309-328.

Broldbent, D. E. Perception and communication. New York: Pergamon Press, 1958.

Darwin, C. J., Turvey, M. T., \& Crowder, R. G. An auditory analogue of the Sperling Partial Report procedure: Evidence for brief auditory storage. Cognitive Psychology, 1972, 3, 255-267.

EstEs, W. K. Interactions of signal and background variables in visual processing. Perception \& Psychophysics, 1972, 12, 278-286.

Garner, W., \& Morton, J. Perceptual independence: Definitions, models, and experimental paradigms. Psychological Bulletin, 1969, 72, 233-258.

Gescheider, G. A., SAger, L. C., \& Ruffolo, L. J. Simultaneous auditory and tactile information processing. Perception \& Psychophysics, 1975, 18, 209-216.

Gilliom, J. D., \& MrLls, W. M. Two-channel detection of the missing event. Joumal of the Acoustical Society of America, 1974, 55, 519. (Abstract)

Gilliom, J. D., \& Sorrn, R. D. Sequential versus simultaneous two-channel signal detection: More evidence for a high-level interrupt theory. Joumal of the Acoustical Society of America, $1974,56,157-164$.

GREEN, D. Detection of auditory sinusoids of uncertain frequency. Journal of the Acoustical Society of America, 1961, 33, 897-903.

KinchlA, R. A. Selective processes in sensory memory: A probecomparison procedure. In S. Kornblum (Ed.), Attention and performance IV. New York: Academic, 1973. Pp. 87-99.

LAPPIN, J. S. Attention in the identification of stimuli in complex visual displays. Joumal of Experimental Psychology, 1967, 75, 321-328.

LindSAY, P. H. Multichannel processing in perception. In D. E. Mostofsky (Ed.), Attention: Contemporary theory and analysis. New York: Appleton-Century-Crofts, 1970. Pp. 149-171.

Lindsay, P. H., Taylor, M. M., \& Forbes, S. M. Attention and multidimensional discrimination. Perception \& Psychophysics, $1968,4,113-117$.

McNicol. D. Feedback as a source of information and as a source of noise in absolute judgments of loudness. Journal of Experimental Psychology: Human Perception and Performance, 1975, 1. 175-182. 
MorAy, N. Attention in dichotic listening: Affective cues and the influence of instructions. Quarterly Journal of Experimental Psychology, 1959, 11, 56-60.

Moray, N. Where is capacity limited? A survey and a model. Acta Psychologica, 1967, 27, 84-93.

MORAY, N. Introductory experiments in auditory time sharing: Detection of intensity and frequency increments. Journal of the Acoustical Society of A merica, 1970, 47, 1071-1073.

Moray, N. A data base for theories of selective listening. In P. M. A. Rabbitt, \& S. Dornic (Eds.), Attention and performance $V$. New York: Academic, 1975. Pp. 119-135.

Moray, N., Bates, A., \& Barnett, T. Experiments on the four eared man. Journal of the Acoustical Society of America, 1965, 38, 196-201.

Norman, D. A., \& Bozrow, D. B. On data-limited and resourcelimited process. Cognitive Psychology, 1975, 7, 44-64.

Parkinson, S. R., Knight, M. V., DeMalo, J. C., \& Connors, J. F. Channels and order of report in dichotic memory. Perception \& Psychophysics, 1974, 16, 235-241.

Pastore, R. E., \& Sorkin, R. D. Simultaneous two-channel signal detection. I. Simple binaural stimuli. Joumal of the Acoustical Society of A merica, 1972, 51, 544-551.

Shiffrin, R. M. \& Grantham, D. W. Can attention be allocated to sensory modalities? Perception \& Psychophysics, 1974, 15, 460-474.

Sorkin, R. D., Pastore, R. E., \& Pohlmann, L. D. Simultaneous two-channel detection. Il. Correlated and uncorrelated signals. Joumal of the Acoustical Society of America, 1972. 51, 1960-1965.

Sorkin, R. D., \& Pohlmann, L. D. Some models of observer behavior in two-channel auditory signal detection. Perception \& Psychophysics, 1973, 14, 101-109.

Sorkin, R. D. . Pohlmann, L. D. \& Gilliom, J. D. Simultaneous two-channel signal detection. III. 630 and $1400 \mathrm{~Hz}$ signals. Journal of the Acoustical Society of America, 1973, 53, 1045-1050.

Sorkin. R. D., Pohlmane, L. D., \& Woods, D. D. Decision interaction between auditory channels. Perception \& Psychophysics, 1976, 19, 290-295.
SPERLING, G. The information available in brief visual presentations. Psychological Monographs, 1960, 74(Whole No. 11).

Treisman, A.. \& Geffen, G. Selective attention: Perception or response. Quarterly Journal of Experimental Psychology, 1967, 19. 1-17.

Treisman, M. Detection of binaural tone stimuli: Time sharing or criterion change. Joumal of the Acoustical Society of America, 1972. 51. 625-630.

Treisman, M., \& Rostron, A. B. Brief auditory storage: A modification of Sperling's paradigm applied to audition. Acta Psychologica. 1972, 36, 161-170.

Wolford, G., \& Hollingsworth, S. Evidence that short-term memory is not the limiting factor in the tachistoscopic full report procedure. Memory \& Cognition, 1974, 2, 796-800.

\section{NOTES}

1. The data reported were obtained by computing d's for the conditional data for each day and then averaging these d's across days. While summing the raw data across days prior to computing $d$ ' will also serve to increase the size of the conditional data cell entries, such a procedure does not allow assessment of the reliability of the conditional effects or of any systematic change in these effects with practice, both of which are of interest. When a particular conditional data matrix contained a zero entry, this conditional data was not included in the reported data. This occurred only rarely for the order of report data, not at all for the stimulus-response conditional data, and frequently when partitioning on both stimulus-response and order of report. Regarding the order of report data, note that an observer may vary the hit rate for a particular channel somewhat independently of the false alarm rate by varying the order of his responses. While imposing a particular response order avoids this problem, it also may significantly change the processing requirements of the task.

(Received for publication December 8, 1975; revision accepted July $21,1976$. ) 